\title{
Effectiveness of 23-Valent Pneumococcal Polysaccharide Vaccine against Invasive Pneumococcal Disease in Adults, Japan, 2013-2017
}

Reiko Shimbashi, Motoi Suzuki, Bin Chang, Hiroshi Watanabe, Yoshinari Tanabe, Koji Kuronuma, Kengo Oshima, Takaya Maruyama, Hiroaki Takeda, Kei Kasahara, Jiro Fujita, Junichiro Nishi, Tetsuya Kubota, Keiko Tanaka-Taya, Tamano Matsui, Tomimasa Sunagawa, Kazunori Oishi, Adult IPD Study Group

The decline in the proportion of pneumococcal conjugate vaccine $(\mathrm{PCV})$-covered serotypes among adult invasive pneumococcal disease (IPD) patients might change the overall effectiveness of the 23-valent pneumococcal polysaccharide vaccine (PPSV23) because its effectiveness differs according to serotype. Using the indirect cohort method, we calculated the effectiveness of PPSV23 against IPD among adults in Japan to assess the impact of the national pediatric PCV program. Clinical and epidemiologic information and pneumococcal isolates were collected from IPD patients $\geq 20$ years of age through enhanced IPD surveillance during April 2013-December 2017. Adjusted effectiveness against PPSV23-serotype IPD was $42.2 \%$. Despite a substantial decline in the proportion of 13-valent PCV serotypes during the study period $(45 \%$ to $31 \%)$, the change in effectiveness for PPSV23-serotype IPD was limited (47.1\% to $39.3 \%)$ and only marginal in the elderly population $(39.9 \%$ to $39.4 \%$ ). The pediatric PCV program had limited impact on PPSV23 effectiveness against IPD in adults.

Streptococcus pneumoniae is a major cause of illness $\mathcal{S}$ and death among adults (1). Pneumonia is the most common form of pneumococcal disease in adults, whereas invasive pneumococcal disease (IPD), including meningitis and bacteremia, has severe clinical manifestations with a high case-fatality ratio (2).
Because incidence of adult IPD is high among older adults, it is a public health concern, particularly in countries with aging populations, such as Japan $(3,4)$.

Two types of pneumococcal vaccines are currently available for adults: the 23-valent pneumococcal polysaccharide vaccine (PPSV23) and the 13-valent pneumococcal conjugate vaccine (PCV13). According to a systematic review, the effectiveness of PPSV23 against IPD among adults $\geq 50$ years of age was $54 \%$ (5). The CAPITA trial showed that the efficacy of PCV13 against IPD among adults $\geq 65$ years of age was 75\% (6). Since 2014, both PPSV23 and PCV13 have been recommended for older persons in the United States (7), whereas only PPSV23 is recommended in other high-income countries.

Discussions over adult pneumococcal vaccination programs are complicated because of differences in vaccine effectiveness (VE) by serotype and age group. Previous studies have suggested that VE of PPSV23 differs by serotype $(8,9)$, so overall VE might vary on the basis of the distribution of serotypes among the vaccinated population. In many countries, the proportion of PCV-covered serotypes among adult IPD patients has been decreasing since the introduction of pediatric PCVs (10-13); this
Author affiliations: National Institute of Infectious Diseases, Tokyo, Japan (R. Shimbashi, M. Suzuki, B. Chang, K. Tanaka-Taya, T. Matsui, T. Sunagawa, K. Oishi); Tohoku University Graduate School of Medicine, Miyagi, Japan (R. Shimbashi, M. Suzuki, K. Oshima); Kurume University School of Medicine, Fukuoka, Japan (H. Watanabe); Niigata Prefectural Shibata Hospital, Niigata, Japan (Y. Tanabe); Sapporo Medical University School of Medicine, Hokkaido, Japan (K. Kuronuma); National Hospital Organization Mie National Hospital, Mie, Japan (T. Maruyama);
Yamagata Saisei Hospital, Yamagata, Japan (H. Takeda); Nara Medical University, Nara, Japan (K. Kasahara); Graduate School of Medicine, University of the Ryukyus, Okinawa, Japan (J. Fujita); Kagoshima University Graduate School of Medical and Dental Sciences, Kagoshima, Japan (J. Nishi); Kochi Medical School, Kochi University, Kochi, Japan (T. Kubota); Toyama Institute of Health, Toyama, Japan (K. Oishi) 
decline might have changed the effectiveness of PPSV23 against PPSV23-serotype IPD. However, the effect of pediatric PCV programs on VE of PPSV23 among adults has not been fully established. In addition, a few studies have suggested that VE is lower among older adults, but evidence is limited $(9,14)$.

In Japan, PCV7 was licensed in November 2010, included in the routine immunization program for children in April 2013, and replaced by PCV13 in October 2013; PPSV23 was included in the routine immunization program for adults $\geq 65$ years of age in 2014. We conducted this study to investigate whether VE of PPSV23 against IPD among adults $\geq 20$ years of age was affected by the pediatric PCV program. We assessed the change in the proportion of PCV-covered serotypes among adult IPD patients across the study period and that in overall VE. We also explored the differences in VE according to age group and other population characteristics.

\section{Methods}

\section{Study Population}

Under the revised Infectious Disease Control Law, national IPD surveillance was implemented in Japan in 2013. Since then, physicians have been required to report all IPD patients and their basic information to the nearest local public health centers. To collect more detailed clinical and epidemiologic information and $S$. pneumoniae isolates from adult IPD patients, in April 2013 the Adult IPD Study Group initiated enhanced surveillance covering 10 of the 47 prefectures of Japan (15). Details of the study design and methods have been described elsewhere $(15,16)$. In brief, all IPD patients $\geq 15$ years of age who had been identified in the local health centers were recruited for enhanced surveillance, and their clinical and epidemiologic information, including PPSV23 vaccination history, was collected by research collaborators by using a standardized case form. S. pneumoniae isolates and clinical specimens were collected from hospital laboratories or prefectural public health institutes and transferred to the National Institute of Infectious Diseases for further testing. A patient was defined as having IPD if the culture was positive for $S$. pneumoniae or if $S$. pneumoniae-specific DNA (lytA gene) was detected by PCR assay in samples collected from normally sterile sites, such as blood and cerebrospinal fluid. To investigate the effectiveness of PPSV23 among adults, we excluded IPD patients $\geq 15-19$ years in this study. IPD patients $\geq 20$ years of age who had been enrolled in the study during April 2013-December 2017 and whose clinical and epidemiologic information and microbiologic testing results were available were included in the analyses.

\section{Microbiological Testing}

S. pneumoniae isolates were serotyped by using the capsule Quellung reaction with rabbit antisera (Statens Serum Institute, https:/ / en.ssi.dk) after culturing overnight. Clinical specimens were serotyped by using the multiplex serotyping PCR assay as described previously $(15,17)$. Because the Quellung reaction could not distinguish between serotypes $11 \mathrm{~A}$ and $11 \mathrm{E}$, these serotypes were grouped into serogroup 11A/E and considered to be the PPSV23 serotype. Isolates that did not react with any antiserum were classified as nontypeable. One isolate per patient was included in our analysis.

\section{Pneumococcal Vaccination Policy in Japan}

In Japan, PPSV23 was included in the national immunization program in October 2014 for all persons $\geq 65$ years of age and those 60-65 years of age with underlying diseases, such as heart disease, kidney disease, respiratory disease, and immunocompromised condition attributable to HIV infection. The cost of vaccination is partly subsidized by the local government. A national catch-up campaign targeting persons $\geq 65$ years of age also was launched in 2014. For persons 2-59 years of age with high-risk conditions (e.g., a history of splenectomy), the cost of vaccination is covered by health insurance. According to an estimate by Japan's Ministry of Health, Labor, and Welfare, $33 \%-38 \%$ of persons $\geq 65$ years of age were vaccinated with PPSV23 during 2014-2017 (18). In our study, PPSV23 vaccination status was obtained from medical records and confirmed by patients or their guardians. Patients were considered vaccinated only if they had received $\geq 1$ dose of PPSV23 in the 5 years before the hospital visit.

PCV7 was approved for the voluntary vaccination of children in February 2010, included in the routine immunization program for children in April 2013, and replaced with PCV13 in November 2013. The coverage rate of the third dose of PCV13 among children was 98\% in 2017 (18). PCV13 was approved for adults $\geq 65$ years of age in June 2014, but its coverage rate among this age group remains very low $(<1 \%)$. In our study, only 4 of 1,121 patients reported having been vaccinated with PCV13.

\section{Procedures}

VE of PPSV23 against IPD was calculated by using an indirect cohort method (Broome's method). The indirect cohort method is a case-control design and has been used to estimate VE of pneumococcal vaccines by 
using pneumococcal disease surveillance data $(9,19)$. In our study, a case was defined as illness in a patient with IPD caused by a PPSV23 serotype (PPSV23-serotype IPD), including serotypes $1,2,3,4,5,6 \mathrm{~B}, 7 \mathrm{~F}, 8,9 \mathrm{~N}$, $9 \mathrm{~V}, 10 \mathrm{~A}, 11 \mathrm{~A}, 12 \mathrm{~F}, 14,15 \mathrm{~B}, 17 \mathrm{~F}, 18 \mathrm{C}, 19 \mathrm{~A}, 19 \mathrm{~F}, 20,22 \mathrm{~F}$, $23 \mathrm{~F}$, and 33F; a control was defined as a patient with IPD caused by a non-PPSV23 serotypes. For the serotype-specific VE estimates, a case was defined as illness in patients with IPD caused by a specific serotype, and a control was defined as a patient with IPD caused by a non-PPSV23 serotype. We compared the odds of PPSV23 vaccination history in cases and controls and calculated VE as (1 - odds ratio) $\times 100 \%$.

The patients were classified into 4 age groups: 20-39, 40-64, 65-79, and 80 years of age. Patients were considered to have immunocompromised conditions if they had any of the following conditions: a history of splenectomy, transplantation, asplenia or hyposplenia, HIV infection, malignancy, autoimmune disease, and complement deficiency $(20,21)$. The patients' body mass index (BMI) values were grouped as low $\left(<18.5 \mathrm{~kg} / \mathrm{m}^{2}\right)$, normal $\left(18.5-24.9 \mathrm{~kg} / \mathrm{m}^{2}\right)$, and high $\left(\geq 25 \mathrm{~kg} / \mathrm{m}^{2}\right)$. Clinical manifestations in IPD patients were classified as pneumonia, meningitis, bacteremia, and other conditions according to the physicians' report. Because the IPD incidence is higher in the autumn and winter seasons compared with other seasons (22), we defined a high season (epidemiologic weeks 1-22 and 49-52) and a low season (epidemiologic weeks 23-48). To assess the effect of the pediatric PCV program, we divided the study period into 2 phases according to the year of diagnosis: the first phase (2013-2015) and the second phase (2016-2017).

\section{Statistical Analyses}

The characteristics of cases and controls were compared by using the $\chi^{2}$ test or Fisher exact test, as appropriate. We used logistic regression models to estimate VE. Because sex, age, study site, year of diagnosis, season, BMI group, presence of an underlying condition, and smoking status were deemed to be potential confounders on the basis of prior knowledge (9), all factors were included in the final multiple logistic regression models. CIs were adjusted for clustering at the local health center level by using robust SEs.

We estimated VE of IPD for PPSV23 serotypes, PCV13 serotypes excluding 6A (PCV13 non-6A serotype), including serotypes 1, 3, 4, 5, 6B, 7F, 9V, 14, 18C, 19A, 19F, and 23F, and PPSV23 serotypes excluding PCV13 serotypes (PPSV23 non-PCV13 serotypes). The serotype-specific VE was estimated for each serotype if its number of isolates was $>30$. To explore the potential effect of cross-immunity produced by PPSV23 on serogroup 6, VE of IPD for serotypes $6 \mathrm{~A}, 6 \mathrm{~B}, 6 \mathrm{C}$, and $6 \mathrm{D}$ was calculated excluding these serotypes from the controls. VE was stratified by the 2 study phases (2013-2015 and 2016-2017). We also conducted stratified analyses to investigate the potential effect of modifications by sex, age group (persons $<65$ years of age and those $\geq 65$ years of age), the presence of underlying conditions, BMI group, and clinical manifestations. The stratum-specific estimates of VE were compared by using a Wald test (test for interaction).

PPSV23 vaccination history was not documented for $23 \%$ of our patients. This group was coded as no record and included in our primary analyses. In a sensitivity analysis, this group was considered to have missing data, and multiple imputations were performed. All analyses were performed by using Stata version 15 (StataCorp, https:/ / www.stata.com).

\section{Ethics}

This study was approved by the Ethics Committee of the National Institute of Infectious Diseases (approval no. 707) and conducted according to the principles expressed in the Declaration of Helsinki. The requirement for obtaining informed consent from all participants was waived because the data do not contain any patient identifiers, and samples were taken as part of standard patient care.

\section{Results}

During the study period (April 2013-December 2017), a total of 1,824 IPD patients $\geq 20$ years of age were identified through the national IPD surveillance program in the study prefectures (24). Among them, 1,138 patients were enrolled in the study (Appendix Figure, https:/ / wwwnc.cdc.gov/EID/article/26/10/19-1531-App1. pdf). S. pneumoniae isolates or clinical specimens were not available for 15 patients, and clinical and epidemiologic data were not available for an additional 2 patients. After excluding these patients, 1,121 patients were eligible for our analyses. S. pneumoniae was identified in 1,117 patients (99.6\%), and S. pneumoniae-specific DNA was detected in 4 patients $(0.4 \%)$.

The characteristics of the 1,121 IPD patients are summarized in Table 1. A total of $679(61 \%)$ patients were men, and the median age was 70 years (range 22-103 years). Among all IPD patients, 746 (66.5\%) were classified as having PPSV23-serotype IPD and $375(33.5 \%)$ were classified as having non-PPSV23serotype IPD. PPSV23-serotype IPD patients less frequently had immunocompromised conditions and more frequently had pneumonia compared with non-PPSV23-serotype IPD patients; otherwise, characteristics were similar between the 2 groups. 
Table 1. Characteristics of 1,121 invasive pneumococcal disease patients with and without PPSV23 serotype, Japan, 2013-2017*

\begin{tabular}{|c|c|c|c|c|}
\hline Characteristic & Total & PPSV23 serotype, $n=746$ & Non-PPSV23 serotype, $n=375$ & $p$ value \\
\hline \multicolumn{5}{|l|}{ Sex } \\
\hline$M$ & $679(61)$ & $443(59)$ & $236(63)$ & \multirow[t]{2}{*}{0.251} \\
\hline $\mathrm{F}$ & $442(39)$ & $303(41)$ & 139 (37) & \\
\hline \multicolumn{5}{|l|}{ Age group, y } \\
\hline $20-39$ & $55(5)$ & $34(5)$ & $21(6)$ & \multirow[t]{4}{*}{0.437} \\
\hline $40-64$ & $309(28)$ & $211(28)$ & $98(26)$ & \\
\hline $65-79$ & $427(38)$ & 291 (39) & $136(36)$ & \\
\hline$\geq 80$ & $330(29)$ & $210(28)$ & $120(32$ & \\
\hline \multicolumn{5}{|l|}{ Study site, prefecture } \\
\hline Hokkaido & $138(12)$ & $85(11)$ & $53(14)$ & \multirow[t]{10}{*}{0.612} \\
\hline Miyagi & $133(12)$ & $92(12)$ & $41(11)$ & \\
\hline Yamagata & $95(8)$ & $69(9)$ & $26(7)$ & \\
\hline Niigata & $211(19)$ & 144 (19) & 67 (18) & \\
\hline Mie & $113(10)$ & $78(10)$ & $35(9)$ & \\
\hline Nara & $80(7)$ & $51(7)$ & $29(8)$ & \\
\hline Kochi & $38(3)$ & $27(4)$ & $11(3)$ & \\
\hline Fukuoka & $222(20)$ & $146(20)$ & $76(20)$ & \\
\hline Kagoshima & $45(4)$ & $28(4)$ & $17(5)$ & \\
\hline Okinawa & $46(4)$ & $26(3)$ & $20(5)$ & \\
\hline \multicolumn{5}{|l|}{ Year } \\
\hline 2013 & $45(4)$ & $33(4)$ & $12(3)$ & \multirow[t]{6}{*}{0.602} \\
\hline 2014 & $201(18)$ & $134(18)$ & $67(18)$ & \\
\hline 2015 & $213(19)$ & $146(20)$ & $67(18)$ & \\
\hline 2016 & $286(26)$ & $180(24)$ & $106(28)$ & \\
\hline 2017 & $363(32)$ & $243(33)$ & $120(32)$ & \\
\hline Unknown & $13(1)$ & $10(1)$ & $3(1)$ & \\
\hline \multicolumn{5}{|l|}{ Season† } \\
\hline High season & $722(64)$ & $492(66)$ & $230(61)$ & \multirow[t]{3}{*}{0.152} \\
\hline Low season & $385(34)$ & $243(33)$ & $142(38)$ & \\
\hline Unknown & $14(1)$ & $11(1)$ & $3(1)$ & \\
\hline \multicolumn{5}{|l|}{ BMI group, $\mathrm{kg} / \mathrm{m}^{2}$} \\
\hline$<18.5$ & $257(23)$ & $171(23)$ & $86(23)$ & \multirow[t]{4}{*}{0.895} \\
\hline $18.5-24.9$ & $526(47)$ & $346(46)$ & $180(48)$ & \\
\hline$\geq 25$ & $167(15)$ & $111(15)$ & $56(15)$ & \\
\hline Unknown & $171(15)$ & $118(16)$ & $53(14)$ & \\
\hline \multicolumn{5}{|l|}{ Underlying conditions } \\
\hline Immunocompromised conditions & $314(28)$ & $175(23)$ & $139(37)$ & \multirow[t]{4}{*}{$<0.001$} \\
\hline Other conditions & $479(43)$ & $324(43)$ & $155(41)$ & \\
\hline Without underlying conditions & $256(23)$ & $198(27)$ & $58(15)$ & \\
\hline Unknown & $72(6)$ & $49(7)$ & $23(6)$ & \\
\hline \multicolumn{5}{|l|}{ Smoking history } \\
\hline Yes & $390(35)$ & $265(36)$ & $125(33)$ & \multirow[t]{3}{*}{0.709} \\
\hline No & $559(50)$ & $370(50)$ & $189(50)$ & \\
\hline Unknown & $172(15)$ & $111(15)$ & $61(16)$ & \\
\hline Alcohol intake & & & & \\
\hline Yes & $184(16)$ & $121(16)$ & $63(17)$ & 0.439 \\
\hline No & $750(67)$ & $493(66)$ & 257 (69) & \\
\hline Unknown & $187(17)$ & $132(18)$ & $55(15)$ & \\
\hline Clinical manifestations & & & & \\
\hline Pneumonia & $665(59)$ & $480(64)$ & $185(49)$ & $<0.001$ \\
\hline Meningitis & 169 (15) & $94(13)$ & $75(20)$ & \\
\hline Bacteremia & $188(17)$ & $104(14)$ & $84(22)$ & \\
\hline Otherł & $98(9)$ & $68(9)$ & $30(8)$ & \\
\hline Unknown & $1(0)$ & $0(0)$ & $1(0)$ & \\
\hline Fatal outcome & & & & \\
\hline Yes & $204(18)$ & $137(18)$ & $67(18)$ & 0.838 \\
\hline No & $917(82)$ & $609(82)$ & $308(82)$ & \\
\hline PPSV23 vaccination within $5 \mathrm{y}$ & & & & \\
\hline Yes & $103(9)$ & $58(8)$ & $45(12)$ & $<0.001$ \\
\hline No & $765(68)$ & $539(72)$ & $226(60)$ & \\
\hline Unknown & $253(23)$ & $149(20)$ & $104(28)$ & \\
\hline
\end{tabular}

*Values are no. (\%) unless indicated. BMI, body mass index; EW, epidemiologic week; PPSV23, 23-valent pneumococcal polysaccharide vaccine. tHigh season indicates the period from EW 1 to EW 22 and from EW 49 to EW 52, whereas low season indicates the period from EW 23 to EW 48. łlncludes arthritis, endocarditis, sinusitis, otitis media, vertebritis, cholecystitis, aortic aneurysm, and pleurisy. 
After we controlled for confounders, VE against PPSV23-serotype IPD was $42.2 \%$ (95\% CI 13.4 to 61.4 ) (Table 2). VE against PCV13 non-6A-serotype IPD was $35.3 \%$ (95\% CI $-8.4 \%$ to $61.5 \%)$ and that against PPSV23 non-PCV13-serotype IPD was 44.5\% (95\% CI $9.6 \%$ to $65.9 \%$ ). Sensitivity analyses showed similar VE estimates (Appendix Table 1). A high level of effectiveness was observed for serotypes 19A (70.3\% [95\% CI $13.3 \%$ to $89.8 \%$ ]), $12 \mathrm{~F}$ (70.8\% [95\% CI $1.0 \%$ to $91.4 \%]$ ), and $10 \mathrm{~A}(73.6 \%$ [95\% CI $5.9 \%$ to $92.6 \%])$. Lowto-moderate effectiveness was observed for serotypes 3 (34.1\% [95\% CI $-34.4 \%$ to $67.7 \%]), 22 \mathrm{~F}(22.7 \%$ [95\% CI $-88.8 \%$ to $68.4 \%]), 11 \mathrm{~A} / \mathrm{E}(20.7 \%$ [95\% CI $-145.4 \%$ to $74.4 \%]$ ), and $7 \mathrm{~F}$ (22.4\% [95\% CI $-176.8 \%$ to $78.2 \%]$ ); however, their CIs were wide because of the limited sample size.

The trend in the proportion of vaccine-covered serotypes among adult IPD patients during the study period is shown in the Figure. The proportion of PCV13 serotypes was $45 \%$ in the first phase of the study (2013-2015) and 31\% in the second phase (2016-2017). When we stratified the patients by age group, the decline was $24 \%(41 \%$ in the first phase and $17 \%$ in the second phase) among patients $20-64$ years of age and $10 \%$ (47\% in the first phase and $37 \%$ in the second phase) among those $\geq 65$ years of age.

VE against PPSV23-serotype IPD among persons $\geq 20$ years of age in the first phase was $47.1 \%$ (95\% CI $-4.7 \%$ to $73.3 \%$ ) and in the second phase was $39.3 \%$ (95\% CI $-2.9 \%$ to $64.2 \%$ ) ( $p=0.953$ by test for interaction) (Table 3). When we focused on persons $\geq 65$ years of age, VE point estimates in the 2 phases showed almost identical values $(39.9 \%$ in the first phase and $39.4 \%$ in the second; $p=0.809$ ). For persons 20-64 years of age, VE point estimates showed a decreasing trend $(77.1 \%$ in the first phase and $41.0 \%$ in the second; $p=0.124)$; however, the CIs were wide.

Our subgroup analyses showed that VE against PPSV23-serotype IPD was $59.0 \%$ (95\% CI $17.9 \%$ to $79.6 \%$ ) among persons 20-64 years of age and 39.2\%
(95\% CI $2.0 \%$ to $62.2 \%$ ) among those $\geq 65$ years of age, but this difference was not statistically significant level ( $p=0.17$ by test for interaction) (Table 4). When we stratified the age group further, VE was $44.6 \%$ (95\% CI $-14.5 \%$ to $73.2 \%$ ) among persons $65-79$ years of age and $31.3 \%$ (95\% CI $-47.7 \%$ to $68.1 \%$ ) among those $\geq 80$ years of age. Higher VE was observed in persons with a normal BMI (70.6\% [95\% CI $47.7 \%$ to $83.5 \%]$ ) than in those with a low BMI (7.4\% [95\% CI -108.1\% to 58.8\%]) or a high BMI (-136.5\% [95\% CI $-826.6 \%$ to $39.6 \%])$. VE did not differ by patients' underlying diseases. Among persons $\geq 65$ years of age, VE for IPD with pneumonia was $52.8 \%$ (95\% CI $16.5 \%$ to $73.3 \%$ ) and for IPD without pneumonia was $9.8 \%$ (95\% CI $-134.4 \%$ to $65.3 \%)$ (Appendix Table 2). Among persons 20-64 years of age, VE for IPD with pneumonia was $23.0 \%$ (95\% CI $-272.2 \%$ to $84.1 \%$ ) and for IPD without pneumonia was $88.8 \%$ (95\% CI $-12.0 \%$ to $98.9 \%)$.

\section{Discussion}

VE of PPSV23 against PPSV23-serotype IPD was 42.2\% among adults $\geq 20$ years of age in Japan. VE against PCV13 non-6A-serotype IPD and that against PPSV23 non-PCV13-serotype IPD were almost comparable. Despite an observed reduction in the proportion of PCV13 serotypes among adult IPD patients during the study period, the change in VE against PPSV23-serotype IPD was limited among adults $\geq 20$ years of age and only marginal among those $\geq 65$ years of age.

Large declines in the incidence of adult pneumococcal disease caused by PCV serotypes have been reported in many countries because of the indirect effect of pediatric PCVs $(10,25-27)$. A pooled analysis of 10 countries in Europe demonstrated that during 20092015, incidence of PCV7-serotype IPD among adults $\geq 65$ years of age declined by $77 \%$ and incidence of PCV 13 non-PCV7-serotype IPD among the same age group declined 38\% (26). The indirect effect of pediatric PCVs is particularly important when making adult pneumococcal vaccination policies because it might affect

\begin{tabular}{|c|c|c|c|c|}
\hline Serotype & No. cases & No. controls & Crude VE, \% (95\% Cl) & Adjusted VE,† \% $(95 \% \mathrm{Cl})$ \\
\hline PPSV23 serotype & 746 & 375 & 46.0 (17.8 to 64.5$)$ & $42.2(13.4$ to 61.4$)$ \\
\hline PCV13, non-6A serotype & 392 & 375 & 40.6 (3.8 to 63.3$)$ & $35.3(-8.4$ to 61.5$)$ \\
\hline PPSV23, non-PCV13 serotype & 354 & 375 & 51.7 (18.7 to 71.3 ) & $44.5(9.6$ to 65.9$)$ \\
\hline Serotype 3 & 152 & 375 & $43.1(-11.9$ to 71.1$)$ & $34.1(-34.4$ to 67.7$)$ \\
\hline Serotype 19A & 111 & 375 & 72.7 (29.1 to 89.5$)$ & $70.3(13.3$ to 89.8$)$ \\
\hline Serotype $12 \mathrm{~F}$ & 99 & 375 & $80.2(34.4$ to 94.0$)$ & $70.8(1.0$ to 91.4$)$ \\
\hline Serotype $22 \mathrm{~F}$ & 83 & 375 & $34.1(-47.1$ to 70.5$)$ & $22.7(-88.8$ to 68.4$)$ \\
\hline Serotype 10A & 80 & 375 & 75.7 (19.2 to 92.7$)$ & 73.6 (5.9 to 92.6$)$ \\
\hline Serotype $11 \mathrm{~A} / \mathrm{E}$ & 41 & 375 & $30.7(-106.7$ to 76.8$)$ & $20.7(-145.4$ to 74.4$)$ \\
\hline Serotype 7F & 30 & 375 & $31.5(-138.6$ to 80.3$)$ & $22.4(-176.8$ to 78.2$)$ \\
\hline
\end{tabular}

*BMI, body mass index; PCV13, 13-valent pneumococcal conjugate vaccine; PPSV23, 23-valent pneumococcal polysaccharide vaccine; VE, vaccine effectiveness.

†Adjusted for sex, age, prefecture, year, season, BMI group, underlying conditions, and smoking history with clustering by public health center. 


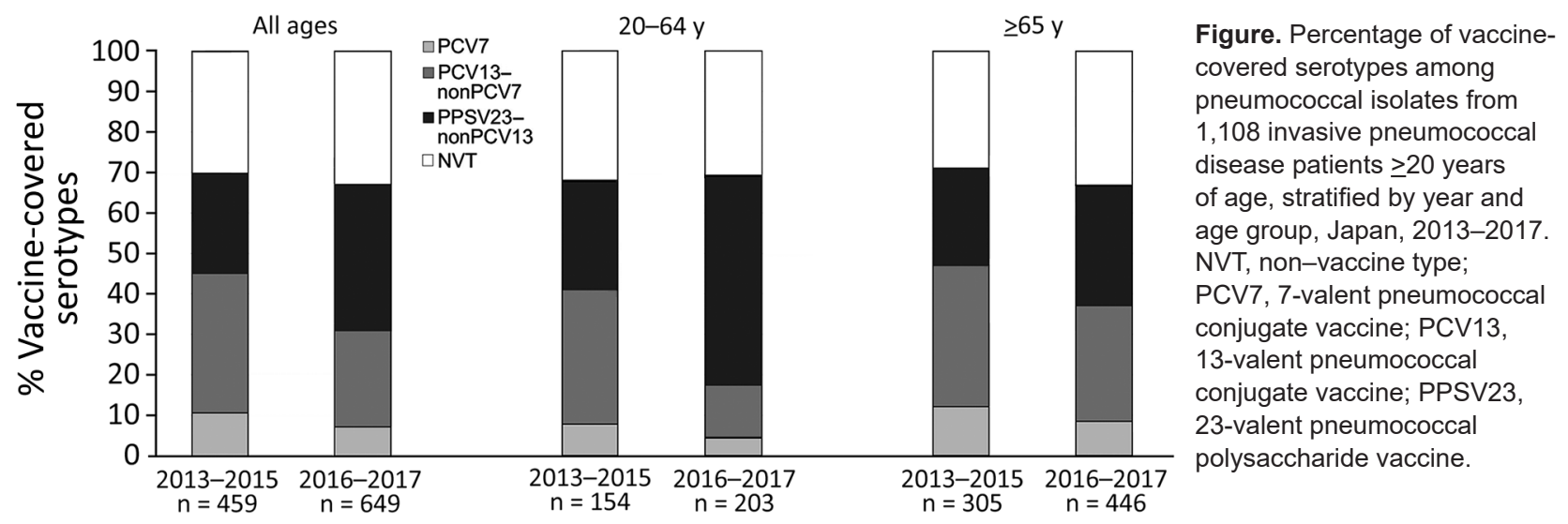

the effectiveness and population impact of adult vaccines. In our study, the proportion of PCV13 serotypes among adult IPD patients decreased from $45 \%$ to $31 \%$, whereas the proportion of PPSV23 non-PCV13 serotypes increased from $25 \%$ to $36 \%$. Because the effectiveness of pneumococcal vaccines is known to differ by serotype, this change in the serotype distribution might have changed overall PPSV23 effectiveness. However, in our study, VE values against PCV13 non-6A-serotype IPD (35.3\% [95\% CI -8.4\% to 61.5\%]) and PPSV23 non-PCV13-serotype IPD (44.5\% [95\% CI $9.6 \%$ to $65.9 \%$ ]) did not differ substantially. Consequently, the change in VE point estimates among adults $\geq 20$ years of age was limited during the study period $(47.1 \%$ in the first phase and $39.3 \%$ in the second), and no change was observed among those $\geq 65$ years of age $(39.9 \%$ in the first phase and $39.4 \%$ in the second). Our findings suggest that VE of PPSV23 among adults in Japan is moderate and remained constant during the 4-year study period under the impact of pediatric PCV13.

In the current study, VE values against IPD varied by serotype; high VE was observed against serotypes 19A, 12F, and 10A, and low-to-moderate VE was observed against serotypes 3, 22F, 11A/E, and $7 \mathrm{~F}$. Serotype 3 was the leading serotype observed in our patients, as has been the case in other countries (28). Studies have shown that the efficacy of pneumococcal vaccines against serotype 3 is limited (28). The observed low-to-moderate VE in our study (34.1\%
[95\% CI $-34.4 \%$ to $67.7 \%$ ]) was consistent with previous studies $(8,9,29)$. Recently, we reported the emergence of serotype 12F among adult IPD patients in Japan (16), and that serotype was the third leading serotype $(9 \%)$ identified in our patients. Increases in incidence of serotype $12 \mathrm{~F}$ have been observed in other countries after the introduction of pediatric PCVs $(30,31)$. Studies have suggested that this serotype is associated with outbreaks and a high invasiveness potential (32-34). The high VE against serotype $12 \mathrm{~F}$ (70.8\% [ $95 \%$ CI 1.0\% to $91.4 \%]$ ) observed in our study suggests that PPSV23 vaccination is an effective measure to reduce its impact.

When we stratified the patients by age group, VE was $59 \%$ (95\% CI $17.9 \%$ to $79.6 \%$ ) for persons $20-64$ years of age and $39.2 \%$ (95\% CI $2 \%$ to $62.2 \%$ ) for those $\geq 65$ years of age; however, this difference was not statistically significant ( $p=0.17$ by test for interaction). A declining trend in PPSV23 effectiveness with age has been reported previously $(9,14)$. A study conducted in Spain showed that VE against IPD was $54.2 \%$ in adults $60-69$ years of age, $54.1 \%$ in adults $70-79$ years of age, and $25.5 \%$ in adults $\geq 80$ years of age (9). These observations might be explained by the decline in pneumococcal polysaccharide immunity with increasing age (35). We found that VE was lower among persons with a low or high BMI than among those with a normal BMI ( $p=0.005$ by test for interaction). Malnutrition, including undernutrition and overnutrition, is known to be associated with immune

\begin{tabular}{|c|c|c|c|}
\hline \multirow[b]{2}{*}{ Age group, y } & \multicolumn{2}{|c|}{ Adjusted VE, $† \%(95 \% \mathrm{Cl})$} & \multirow[b]{2}{*}{$p$ value by test for interaction } \\
\hline & $2013-2015$ & $2016-2017$ & \\
\hline Overall & $47.1(-4.7$ to 73.3$)$ & $39.3(-2.9$ to 64.2$)$ & 0.953 \\
\hline $20-64$ & $77.1(-110.4$ to 97.5$)$ & $41.0(-128.8$ to 84.8$)$ & 0.124 \\
\hline$\geq 65$ & $39.9(-28.4$ to 71.9$)$ & $39.4(-6.1$ to 65.3$)$ & 0.809 \\
\hline
\end{tabular}

${ }^{*} \mathrm{BMI}$, body mass index; PPSV23, 23-valent pneumococcal polysaccharide vaccine; VE, vaccine effectiveness.

†Adjusted for sex, age, prefecture, year, season, BMI group, underlying conditions, and smoking history with clustering by public health center. 
Table 4. Stratified analyses of the effectiveness of PPSV23 against invasive pneumococcal disease in adults $\geq 20$ years of age, Japan, 2013-2017*

\begin{tabular}{|c|c|c|c|c|c|}
\hline Characteristic & No. cases & No. controls & Crude VE, $\%(95 \% \mathrm{Cl})$ & Adjusted VE, $† \%(95 \% \mathrm{Cl})$ & $\mathrm{p}$ value \\
\hline \multicolumn{6}{|l|}{ Sex } \\
\hline M & 443 & 236 & 41.5 (2.0 to 65.0$)$ & $38.7(-5.1$ to 64.3$)$ & \multirow[t]{2}{*}{0.917} \\
\hline $\mathrm{F}$ & 303 & 139 & 52.7 (2.5 to 77.1$)$ & 48.5 (5.3 to 72.0$)$ & \\
\hline \multicolumn{6}{|l|}{ Age group, y } \\
\hline $20-64$ & 245 & 119 & 72.5 (13.6 to 91.3$)$ & 59.0 (17.9 to 79.6$)$ & \multirow{2}{*}{0.170} \\
\hline$\geq 65$ & 501 & 256 & $41.7(7.2$ to 63.3$)$ & $39.2(2.0$ to 62.2$)$ & \\
\hline \multicolumn{6}{|l|}{ Clinical manifestations } \\
\hline Pneumonia & 480 & 185 & 55.8 (26.2 to 73.5$)$ & 50.6 (16.0 to 70.9$)$ & \multirow[t]{4}{*}{0.284} \\
\hline Meningitis & 94 & 75 & $46.9(-76.6$ to 84.1$)$ & $35.6(-100.0$ to 79.2$)$ & \\
\hline Bacteremia & 104 & 84 & $41.8(-78.2$ to 81.0$)$ & $34.7(-72.1$ to 75.2$)$ & \\
\hline Other $\ddagger$ & 68 & 30 & NA & NA & \\
\hline \multicolumn{6}{|l|}{ BMI group, $\mathrm{kg} / \mathrm{m}^{2}$} \\
\hline$<18.5$ & 171 & 86 & $11.3(-109.4$ to 62.4$)$ & $7.4(-108.1$ to 58.8$)$ & \multirow[t]{3}{*}{0.005} \\
\hline 18.5-24.9 & 346 & 180 & 73.2 (50.8 to 85.4$)$ & 70.6 (47.7 to 83.5$)$ & \\
\hline$\geq 25$ & 111 & 56 & $-133.3(-765.0$ to 37.1$)$ & $-136.5(-826.6$ to 39.6$)$ & \\
\hline \multicolumn{6}{|l|}{ Underlying conditions } \\
\hline Immunocompromised & 175 & 139 & $41.0(-22.0$ to 71.4$)$ & $41.2(-27.6$ to 72.9$)$ & \multirow[t]{3}{*}{0.971} \\
\hline Other condition & 324 & 155 & 48.5 (7.2 to 71.4$)$ & $48.2(6.0$ to 71.5$)$ & \\
\hline No underlying condition & 198 & 58 & $48.8(-113.3$ to 87.7$)$ & 51.5 (-116.0 to 89.1$)$ & \\
\hline
\end{tabular}

defects (36) and poor vaccine-induced immune responses (37). On the other hand, VE did not differ between persons with and without underlying conditions. A similar finding was observed in a previous study conducted in Japan; the effectiveness of PPSV23 against pneumococcal pneumonia among adults $\geq 65$ years of age did not differ by their underlying condition status (29). These observations might be at least partially explained by the low prevalence of HIV infection in adults in Japan. Only 1 patient was recorded as being HIV-positive in our study.

VE for bacteremic pneumococcal pneumonia among persons $\geq 65$ years of age was $52.8 \%$ (95\% CI $16.5 \%$ to $73.3 \%$ ), whereas among persons $20-64$ years of age it was $23.0 \%$ ( $95 \%$ CI $-272.2 \%$ to $84.1 \%$ ) (p = 0.064 by test for interaction). Because pneumonia is the most common manifestation of pneumococcal disease among the elderly population (15), this finding might support the current PPSV23 recommendations. On the other hand, although the CI was wide, the VE point estimate for non-pneumonia-associated IPD, such as meningitis and occult bacteremia (i.e., bacteremia without an identifiable focus of infection), was high in the younger age group. The potential difference in PPSV23 effectiveness according to population characteristics and clinical manifestations is particularly important when creating efficient vaccination policies. Further studies are needed to understand the mechanisms underlying our observations.

In Japan, PPSV23 was introduced into the adult immunization program in 2014, but its vaccination coverage rate was only $\approx 30 \%$ in 2017 . Recently, the
Ministry of Health, Labor, and Welfare decided to extend the duration of the catch-up campaign for persons $\geq 65$ years of age until 2023. The continued moderate PPSV23 effectiveness under the impact of the pediatric PCV13 program we observed provides supporting evidence for the current adult pneumococcal vaccination policy. However, making decisions regarding the adult PPSV23 program is still challenging for several reasons, such as its low level of efficacy in the older age group $(8,9)$ and limited evidence supporting repeated vaccinations $(38,39)$. Continuous monitoring of the serotype distribution and VE among adults is warranted. On the other hand, high VE among younger adults, particularly for meningitis and occult bacteremia, might facilitate a discussion regarding the potential expansion of the target age group.

Our study has limitations. First, $37.6 \%$ of the patients identified in the local health centers were not included in our study. The inclusion rate was especially low at the beginning of the study period; however, the baseline characteristics of the patients did not differ between the enrolled and nonenrolled patients (Appendix Table 3). The effect of selection bias on our VE estimates must have been minimal. Second, vaccination history was not documented in $23 \%$ of our patients. Our sensitivity analyses showed almost identical estimates, so we do not believe this shortcoming affects our observations. Third, we used the indirect cohort method, which is equivalent to the test-negative design, to estimate the PPSV23 effectiveness. Although this design is less susceptible to bias associated with confounding by healthcare-seeking behavior, as in the 
nature of observational study design, the bias is unlikely to be eliminated $(40,41)$. However, our VE estimates are comparable with previous estimates resulting from other study designs (5), so the effect of bias is probably minimal. Finally, only a history of PPSV23 vaccination within 5 years was available. Patients who had received the latest PPSV23 $>5$ years before diagnosis were classified as unvaccinated. If VE lasted $>5$ years, our VE estimates might have underestimated actual VEs. Also, our study could not assess the waning of effectiveness over the 5 years.

In conclusion, the effectiveness of PPSV23 against IPD is moderate among adults $\geq 20$ years of age in Japan. Although the proportion of PCV13 serotypes among adult IPD patients has been substantially decreasing because of the indirect effect of the pediatric PCV program, the change in PPSV23 effectiveness was limited.

\section{Acknowledgments}

We sincerely thank the staff of the local public health centers who collected bacterial isolates and case report forms from the hospitals and the staff of the public health institutes and laboratories who worked for the Adult IPD Study Group (http://www.nih.go.jp/niid/ja/ibi.html).

This work was supported by MHLW HA Program Grant Number JPMH20HA1005.

\section{About the Author}

Dr. Shimbashi is a researcher on the field of vaccination at the National Institute of Infectious Diseases in Japan. Her primary research interests are vaccine effectiveness, vaccine adverse events, and vaccine communication strategies.

\section{References}

1. Peto L, Nadjm B, Horby P, Ngan TT, van Doorn R, Van Kinh N, et al. The bacterial aetiology of adult community-acquired pneumonia in Asia: a systematic review. Trans R Soc Trop Med Hyg. 2014;108:326-37. https://doi.org/10.1093/trstmh/tru058

2. Houseman C, Chapman KE, Manley P, Gorton R, Wilson D, Hughes GJ. Decreasing case fatality rate following invasive pneumococcal disease, North East England, 2006-2016. Epidemiol Infect. 2019;147:e175. https:/ / doi.org/10.1017/ S0950268819000657

3. Drijkoningen JJ, Rohde GG. Pneumococcal infection in adults: burden of disease. Clin Microbiol Infect. 2014;20 (Suppl 5):45-51. https:/ / doi.org/10.1111/1469-0691.12461

4. Morimoto K, Suzuki M, Ishifuji T, Yaegashi M, Asoh N, Hamashige N, et al.; Adult Pneumonia Study Group-Japan (APSG-J). The burden and etiology of community-onset pneumonia in the aging Japanese population: a multicenter prospective study. PLoS One. 2015;10:e0122247. https://doi.org/10.1371/journal.pone.0122247

5. Kraicer-Melamed H, O'Donnell S, Quach C. The effectiveness of pneumococcal polysaccharide vaccine 23 (PPV23) in the general population of 50 years of age and older: A systematic review and meta-analysis. Vaccine. 2016;34:1540-50. https://doi.org/10.1016/j.vaccine.2016.02.024

6. Bonten MJ, Huijts SM, Bolkenbaas M, Webber C, Patterson S, Gault S, et al. Polysaccharide conjugate vaccine against pneumococcal pneumonia in adults. N Engl J Med. 2015;372:1114-25. https:/ / doi.org/10.1056/NEJMoa1408544

7. Tomczyk S, Bennett NM, Stoecker C, Gierke R, Moore MR, Whitney CG, et al.; Centers for Disease Control and Prevention (CDC). Use of 13-valent pneumococcal conjugate vaccine and 23-valent pneumococcal polysaccharide vaccine among adults aged $\geq 65$ years: recommendations of the Advisory Committee on Immunization Practices (ACIP). MMWR Morb Mortal Wkly Rep. 2014;63:822-5.

8. Andrews NJ, Waight PA, George RC, Slack MP, Miller E. Impact and effectiveness of 23-valent pneumococcal polysaccharide vaccine against invasive pneumococcal disease in the elderly in England and Wales. Vaccine. 2012;30:6802-8. https:// doi.org/10.1016/j.vaccine.2012.09.019

9. Gutierrez Rodriguez MA, Ordobas Gavin MA, Garcia-Comas L, Sanz Moreno JC, Cordoba Deorador E, Lasheras Carbajo MD, et al. Effectiveness of 23-valent pneumococcal polysaccharide vaccine in adults aged 60 years and over in the Region of Madrid, Spain, 2008-2011. Euro Surveill. 2014;19:20922. https:/ / doi.org/10.2807/ 1560-7917.ES2014.19.40.20922

10. Djennad A, Ramsay ME, Pebody R, Fry NK, Sheppard C, Ladhani SN, et al. Effectiveness of 23-valent polysaccharide pneumococcal vaccine and changes in invasive pneumococcal disease incidence from 2000 to 2017 in those aged 65 and over in England and Wales. EClinicalMedicine. 2019;6:42-50. https:/ / doi.org/10.1016/j.eclinm.2018.12.007

11. Ubukata K, Takata M, Morozumi M, Chiba N, Wajima T, Hanada S, et al.; Invasive Pneumococcal Diseases Surveillance Study Group. Effects of pneumococcal conjugate vaccine on genotypic penicillin resistance and serotype changes, Japan, 2010-2017. Emerg Infect Dis. 2018;24:2010-20. https://doi.org/10.3201/eid2411.180326

12. Naucler P, Galanis I, Morfeldt E, Darenberg J, Örtqvist $\AA$, Henriques-Normark B. Comparison of the impact of pneumococcal conjugate vaccine 10 or pneumococcal conjugate vaccine 13 on invasive pneumococcal disease in equivalent populations. Clin Infect Dis. 2017;65:1780-9. https://doi.org/10.1093/cid/cix685

13. Càmara J, Marimón JM, Cercenado E, Larrosa N, Quesada MD, Fontanals D, et al. Decrease of invasive pneumococcal disease (IPD) in adults after introduction of pneumococcal 13-valent conjugate vaccine in Spain. PLoS One. 2017;12:e0175224. https:// doi.org/10.1371/ journal.pone.0175224

14. Singleton RJ, Butler JC, Bulkow LR, Hurlburt D, O'Brien KL, Doan $\mathrm{W}$, et al. Invasive pneumococcal disease epidemiology and effectiveness of 23-valent pneumococcal polysaccharide vaccine in Alaska native adults. Vaccine. 2007;25:2288-95. https:// doi.org/10.1016/j.vaccine.2006.11.065

15. Fukusumi M, Chang B, Tanabe Y, Oshima K, Maruyama T, Watanabe $\mathrm{H}$, et al.; Adult IPD Study Group. Invasive pneumococcal disease among adults in Japan, April 2013 to March 2015: disease characteristics and serotype distribution. BMC Infect Dis. 2017;17:2. https:/ / doi.org/10.1186/ s12879-016-2113-y

16. Shimbashi R, Chang B, Tanabe $Y$, Takeda H, Watanabe $H$, Kubota T, et al.; Adult IPD Study Group. Epidemiological and clinical features of invasive pneumococcal disease caused by serotype $12 \mathrm{~F}$ in adults, Japan. PLoS One. 2019;14:e0212418. https://doi.org/10.1371/journal. pone. 0212418 
17. da Gloria Carvalho M, Pimenta FC, Jackson D, Roundtree A, Ahmad Y, Millar EV, et al. Revisiting pneumococcal carriage by use of broth enrichment and PCR techniques for enhanced detection of carriage and serotypes. J Clin Microbiol. 2010;48:1611-8. https://doi.org/10.1128/ JCM.02243-09

18. Ministry of Health, Labor, and Welfare. The vaccine coverage in routine immunization program in Japan from 1995 to 2017 [cited 2019 Aug 10]. https:/ / www.mhlw.go.jp/topics/bcg/ other/5.html

19. Broome CV, Facklam RR, Fraser DW. Pneumococcal disease after pneumococcal vaccination: an alternative method to estimate the efficacy of pneumococcal vaccine. N Engl J Med. 1980;303:549-52. https://doi.org/10.1056/ NEJM198009043031003

20. Dominguez A, Salleras L, Fedson DS, Izquierdo C, Ruiz L, Ciruela P, et al. Effectiveness of pneumococcal vaccination for elderly people in Catalonia, Spain: a case-control study. Clin Infect Dis. 2005;40:1250-7. https:/ / doi.org/10.1086/ 429236

21. Jackson LA, Neuzil KM, Yu O, Benson P, Barlow WE, Adams AL, et al.; Vaccine Safety Datalink. Effectiveness of pneumococcal polysaccharide vaccine in older adults. N Engl J Med. 2003;348:1747-55. https:/ / doi.org/10.1056/ NEJMoa022678

22. Weinberger DM, Grant LR, Steiner CA, Weatherholtz R, Santosham M, Viboud C, et al. Seasonal drivers of pneumococcal disease incidence: impact of bacterial carriage and viral activity. Clin Infect Dis. 2014;58:188-94. https://doi.org/10.1093/cid/cit721

23. Kim HW, Lee S, Kim KH. Serotype 6B from a pneumococcal polysaccharide vaccine induces cross-functional antibody responses in adults to serotypes $6 \mathrm{~A}, 6 \mathrm{C}$, and $6 \mathrm{D}$. Medicine (Baltimore). 2016;95:e4854. https:// doi.org/ 10.1097/MD.0000000000004854

24. Infectious Disease Surveillance Center, National Institute of Infectious Diseases. The number of weekly reports of the notifiable diseases [cited 2019 Aug 10]. https:/ / www. niid.go.jp/niid/ja/idwr.html

25. Mackenzie GA, Hill PC, Jeffries DJ, Hossain I, Uchendu U, Ameh D, et al. Effect of the introduction of pneumococcal conjugate vaccination on invasive pneumococcal disease in The Gambia: a population-based surveillance study. Lancet Infect Dis. 2016;16:703-11. https:/ /doi.org/10.1016/ S1473-3099(16)00054-2

26. Hanquet G, Krizova P, Valentiner-Branth P, Ladhani SN, Nuorti JP, Lepoutre A, et al.; SpIDnet/I-MOVE+ Pneumo Group. Effect of childhood pneumococcal conjugate vaccination on invasive disease in older adults of 10 European countries: implications for adult vaccination. Thorax. 2019;74:473-82. https://doi.org/10.1136/thoraxjnl-2018-211767

27. Moore MR, Link-Gelles R, Schaffner W, Lynfield R, Lexau C, Bennett NM, et al. Effect of use of 13-valent pneumococcal conjugate vaccine in children on invasive pneumococcal disease in children and adults in the USA: analysis of multisite, population-based surveillance. Lancet Infect Dis. 2015;15:301-9. https:/ / doi.org/10.1016/ S1473-3099(14)71081-3

28. Linley E, Bell A, Gritzfeld JF, Borrow R. Should pneumococcal serotype 3 be included in serotype-specific immunoassays? Vaccines (Basel). 2019;7:E4. https://doi.org/ 10.3390/vaccines7010004

29. Suzuki M, Dhoubhadel BG, Ishifuji T, Yasunami M, Yaegashi M, Asoh N, et al.; Adult Pneumonia Study GroupJapan (APSG-J). Serotype-specific effectiveness of 23-valent pneumococcal polysaccharide vaccine against pneumococcal pneumonia in adults aged 65 years or older: a multicentre, prospective, test-negative design study. Lancet Infect Dis. 2017;17:313-21. https:// doi.org/10.1016/S1473-3099 (17)30049-X

30. Slotved HC, Dalby T, Hoffmann S. The effect of pneumococcal conjugate vaccines on the incidence of invasive pneumococcal disease caused by ten non-vaccine serotypes in Denmark. Vaccine. 2016;34:769-74. https:// doi.org/10.1016/j.vaccine.2015.12.056

31. Rokney A, Ben-Shimol S, Korenman Z, Porat N, Gorodnitzky Z, Givon-Lavi N, et al. Emergence of Streptococcus pneumoniae serotype $12 \mathrm{~F}$ after sequential introduction of 7 - and 13-valent vaccines, Israel. Emerg Infect Dis. 2018;24:453-61. https:/ / doi.org/10.3201/eid2403.170769

32. Hoge CW, Reichler MR, Dominguez EA, Bremer JC, Mastro TD, Hendricks KA, et al. An epidemic of pneumococcal disease in an overcrowded, inadequately ventilated jail. N Engl J Med. 1994;331:643-8. https://doi.org/ 10.1056/NEJM199409083311004

33. Sandgren A, Sjostrom K, Olsson-Liljequist B, Christensson B, Samuelsson A, Kronvall G, et al. Effect of clonal and serotype-specific properties on the invasive capacity of Streptococcus pneumoniae. J Infect Dis. 2004;189:785-96. https://doi.org/10.1086/381686

34. Schillberg E, Isaac M, Deng X, Peirano G, Wylie JL, Van Caeseele P, et al. Outbreak of invasive Streptococcus pneumoniae serotype $12 \mathrm{~F}$ among a marginalized inner-city population in Winnipeg, Canada, 2009-2011. Clin Infect Dis. 2014;59:651-7. https://doi.org/10.1093/cid/ciu366

35. Adler H, Ferreira DM, Gordon SB, Rylance J. Pneumococcal capsular polysaccharide immunity in the elderly. Clin Vaccine Immunol. 2017;24:e00004-00017. https:/ / doi.org/ 10.1128/CVI.00004-17

36. Bourke CD, Berkley JA, Prendergast AJ. Immune dysfunction as a cause and consequence of malnutrition. Trends Immunol. 2016;37:386-98. https:/ / doi.org/10.1016/ j.it.2016.04.003

37. Painter SD, Ovsyannikova IG, Poland GA. The weight of obesity on the human immune response to vaccination. Vaccine. 2015;33:4422-9. https://doi.org/10.1016/ j.vaccine.2015.06.101

38. Kawakami K, Kishino H, Kanazu S, Takahashi K, Iino T, Sawata M, et al. Time interval of revaccination with 23-valent pneumococcal polysaccharide vaccine more than 5 years does not affect the immunogenicity and safety in the Japanese elderly. Hum Vaccin Immunother. 2018;14:1931-8. https:/ / doi.org/10.1080/21645515.2018.1456611

39. Clutterbuck EA, Lazarus R, Yu LM, Bowman J, Bateman EA, Diggle L, et al. Pneumococcal conjugate and plain polysaccharide vaccines have divergent effects on antigen-specific B cells. J Infect Dis. 2012;205:1408-16. https://doi.org/10.1093/infdis/jis212

40. Sullivan SG, Tchetgen Tchetgen EJ, Cowling BJ. Theoretical basis of the test-negative study design for assessment of influenza vaccine effectiveness. Am J Epidemiol. 2016;184:345-53. https:/ / doi.org/10.1093/aje/kww064

41. Omori R, Cowling BJ, Nishiura H. How is vaccine effectiveness scaled by the transmission dynamics of interacting pathogen strains with cross-protective immunity? PLoS One. 2012;7:e50751. https://doi.org/10.1371/journal.pone.0050751

Address for correspondence: Motoi Suzuki, Infectious Disease Surveillance Center, National Institute of Infectious Diseases, 1-23-1 Toyama, Shinjuku, Tokyo 162-8640, Japan; email: mosuzuki@niid.go.jp 